\title{
Los futuros de la Antropología. Las Antropologías del futuro
}

\author{
The Futures of Anthropology. The Anthropologies of the Future
}

\author{
José PALACIOS RAMíREZ \\ Universidad Católica San Antonio Murcia*
}

\begin{abstract}
Resumen: Este texto tiene como objetivo básico sintetizar las respuestas recolectadas en la encuesta que origina este número de la Revista Murciana de Antropología, en torno a la doble cuestión de la definición de la identidad disciplinar de la Antropología, y de sus perspectivas de futuro. Además, trataré de contextualizar brevemente el tema del cuestionamiento por su futuro en el marco de la historia reciente de la disciplina, y de ofrecer un breve comentario personal al respecto, centrado en el interés de incorporar al conjunto de estrategias actuales la reflexión sobre el futuro de nuestras sociedades.
\end{abstract}

Palabras clave: Identidad disciplinar; futuro de la antropología; encuesta; antropología del futuro.

\begin{abstract}
This text has as basic objective to synthesize the collected answers from the survey which origins this Revista Murciana de Antropología number, on the double issue of the definition of the Anthropology's identity and their future perspectives. Besides that, I will try to contextualize briefly the issue of the discussion about the future in the frame of the recent discipline history. Also try to offer a personal comment about the question focused on the possibility of mix in the core of current strategies the reflection on the future of our societies.
\end{abstract}

Key words: Disciplinary identity; future of anthropology; survey; anthropology of the future.

\footnotetext{
* Recibido: 20, octubre, 2018. Aceptado: 21, noviembre, 2018.

Email: jpalacios@ucam.edu.

http://dx.doi.org/10.6018/rmu/355561
} 


\section{INTRODUCCIÓN}

Declinado de distinto modo, en distintos contextos y por supuesto con diferentes intencionalidades, el cuestionamiento por la «identidad» disciplinar y por sus posibles devenires proyectados en el futuro, ocupa un espacio central en el desarrollo histórico de la Antropología social y cultural. ${ }^{1}$ Tanto en Europa como en América, ya sea en sus configuraciones iniciales como ciencia colonial ligada al evolucionismo y al positivismo, o como disciplina «social» anclada «nacionalmente» en la gestión política de la diversidad. Y así parece que sigue siendo en tiempos de despliegue global y complejización de los paisajes sociales, científicos-tecnológicos y políticos. ${ }^{2}$ En toda esa serie histórica de cuestionamientos reflexivos, que por otro lado dificilmente pueden encontrarse en otra disciplina científica, hay un aspecto estructural que parece repetirse. El cuestionamiento por la definición de la disciplina así como los planteamientos sobre su futuro, se dan siempre en términos de crisis, de amenazas e incluso en ocasiones de peticiones de abandono, de disolución disciplinar. Puede decirse que hasta C. Levi-Strauss, dichos cuestionamientos tienen mucho más que ver con procesos de negociación entre las particularidades de las «escuelas» teórico-metodológicas y la «horma» positivista, de ahí que cada nueva escuela «dominante» emergiera prometiendo la «deseada» conversión de la Antropología en una ciencia normal. ${ }^{3}$ A partir de ahí, sobre todo entre las décadas de los 60 y los 70, los términos de estos cuestionamientos se tornan más graves y se radicalizan en sus críticas al concepto de cultura y a la institucionalización de la disciplina como saber aplicado, a la vez que las condiciones infraestructurales y la presencia social de la disciplina van perdiendo empuje.

La razón de que comience con este algo extenso desvío argumental es doble. En primer lugar trato de hacer ver, de entrada, la complejidad y la importancia que guarda la cuestión que se plantea en el texto y en la encuesta, y la reflexión que da lugar a este número. $\mathrm{Y}$ en segundo lugar, también pretendo mostrar la necesidad de enmarcar las respuestas de la encuesta, que después presentaremos, y la posterior reflexión en algún tipo de relato disciplinar, que obviamente conlleva, para quien escribe estas líneas, un privilegio por verme abocado a escribir sobre

1 Comaroff, J.: «The End of Anthropology, Again: On the Future of an In/Discipline», American Anthropologist, 112 (4), 2010, pp. 524-538.

2 Ong, A.; Collier, S. (eds.): Global Assemblages: Technology, Politics, and Ethics as Anthropological Problems, Nueva York: Blackwell, 2004.

3 Kunn, Thomas: La estructura de las revoluciones cientificas, México DF: Fondo de Cultura Económica, 2004. 
una cuestión tan trascendental, a la vez que algo de vértigo por la precariedad de lo que pueda representar mi posición sobre una cuestión en torno a la cual nada parece estar claro.

En cualquier caso, desde mi posición y de forma general, dicho relato marco diría tal que así. Mientras que en Europa a finales de los 70 el británico Rodney Needham, ${ }^{4}$ uno los representantes de las síntesis entre estructuralismo y funcionalismo, auguraba la destrucción de la disciplina debido a su dispersión temática, subdisciplinar y conceptual; la Antropología estadounidense se hallaba inmersa en un profundo proceso de reconfiguración, iniciada en la década de los 60 y que con muchos matices y cambios se mantendría hasta los 80 , inaugurando un marco de tensiones epistémicas, éticas y políticas para las prácticas y conocimientos disciplinares que se prolonga hasta hoy. ${ }^{5}$ En este sentido, destacaría primeramente la compilación Reinventing Anthropology, ${ }^{6}$ que ponía sobre la mesa el corolario de posiciones críticas relativas al necesario compromiso de la disciplina y de los etnógrafos para con los convencionalmente denominados «grupos de estudio», y también en términos de crítica institucional a los aparatos de intervención estatal y de las lógicas macroeconómicas. Como, por su parte, el conocido Writing Culture $^{7}$ junto a la recapitulación de los efectos del llamado debate posmoderno divulgado a través de la mencionada compilación nacida fruto del tan nombrado Seminario de Santa Fe, que representa Anthropology as a Cultural Critique. ${ }^{8}$ Estos referentes constituyen verdaderos manifiestos relativos a la cuestión de la identidad y el futuro disciplinar en contextos socio-históricos muy concretos, que desde otras prácticas teóricas y etnográficas esbozaban algunas de las características del ecléctico, rico y desigual panorama de las antropologías actuales.

$\mathrm{Y}$ aquí sería necesario hacer un inciso aclaratorio por partida doble. Cabe recordar que en su recapitulación sobre lo que consideraron un verdadero «momento experimental» en la Antropología, y por ende en las ciencias humanas y sociales, G. Marcus y M. Fischer proponían tres ejes temáticos que vendrían a articular las propuestas del panorama producido por los cuestionamientos críticos y agrios debates sobre el estatuto epistémico y político de las representaciones

4 Needham, R.: «The future of Social Anthropology: disintegration or metamorphosis», Anniversary Contributions to Anthropology: twelve essays, Leiden: Brill, 1970.

5 Fernández de Rota y Monter, J. A.: Una etnografia de los antropólogos en EE.UU. Consecuencias de los debates posmodernos, Madrid: Akal, 2012.

6 Hymes, D. (ed.): Reinventing Anthropology, New York: Vintage Books, 1970.

7 Clifford, J.; Marcus, G.: Writing Culture. The Poetics and Politics of Ethnography, Berkeley: University of California Press, 1986.

8 Marcus, G.; Fischer, M.: Anthropology as Cultural Critique. An Experimental Moment in the Human Sciences, Chicago: Chicago University Press, 1986. 
antropológicas sobre los sistemas culturales. ${ }^{9}$ Estos ejes eran la persona, el yo y las emociones, la economía política histórico-mundial, y la crítica cultural. Grosso modo esas eran las nuevas vías a final del siglo pasado para la producción de nuevas antropologías. Para presentar un panorama actualizado, tan solo sería necesario añadir a este conjunto las nuevas formas de conocimiento y práctica antropológica ligadas a las transformaciones sociales en proceso, como la centrada en la ciencia y la tecnología, el diseño, el género, o los nuevos movimientos sociales. No se trata solo de un deslizamiento en los temas, se trata también de la emergencia de nuevas formas de practicar y entender la disciplina. Por otro lado, y en conexión con la cuestión de la práctica, hay que señalar que las posiciones y movimientos de crítica se remontan en esta cuestión a los 60 , y que, visto desde hoy, sus efectos han sido quizá más profundos y duraderos que las críticas de corte epistémico y estético del posmodernismo más interpretativista. Desde entonces puede seguirse una cadena de posicionamientos y llamados a diferentes formas de entendimiento de la práctica antropológica como forma específica de militancia, abogacía o activismo. ${ }^{10}$ Quizá la muestra más clara de este efecto en ámbitos como el nuestro, sería la exuberancia alcanzada por campos antes aplicados en un sentido clásico, como la salud, el desarrollo o la educación, a partir de la emergencia y asentamiento de posicionamientos antropológicos fundamentados en la distinción y el juego con las perspectivas de/para.

Retomando nuestro relato, puede decirse que ya en el fin de siglo y a comienzos del nuevo las perspectivas de futuro no parecían tan negativas, parecían encontrarse equilibrios interesantes entre lo que podríamos llamar tradiciones críticas más convencionales y las cercanas al posmodernismo, y algunos autores $^{11}$ comenzaban a apuntar la idea de que la Antropología no enfrentaba

9 El citado trabajo sintetiza bastante bien, creo, aunque sea desde una percepción muy concreta, el ambivalente panorama de la antropología que emergía a final de siglo XX. A mi entender, la permanencia de la centralidad teórica norteamericana, ahora con un sentido mucho más ecléctico y unas condiciones de producción del conocimiento también distintas; una descentralización de los nodos de prestigio y producción antropológica; y consecuentemente la aparición, además intensificada, de lo que siempre fue la disciplina, aunque no apareciera así, como un conjunto amplio de estrategias teóricas y metodológicas con una serie de grandes cuestiones en común. En resumen, la unidad disciplinar no era algo a conservar sino a negociar.

10 Scheper-Hughes, N.: «The Primacy of the Ethical Propositions for a Militant Anthropology», Current Anthropology 36 (3), 1995, pp. 409-440. Clifford, J.: «Looking in Several Ways: Anthropology and Native Heritage in Alaska», Current Anthropology, 45 (1), 2004, pp. 5-30. EscobAr, A.: «Culture, Practice and Politics: Anthropology and the Study of Social Movements», Critique of Anthropology 12 (4), 1992, pp. 395-432.

11 Akbar, A.; Shore, C. (eds.): The Future of Anthropology: its Relevance to the Contemporary World, Londres: Athlone, 1995. 
tanto una crisis epistemológica, como una necesidad de reconquistar el terreno perdido en la discusión de lo que muchos autores llaman «grandes temas» sobre el ser humano, así como en los debates públicos de las sociedades sobre los problemas y su resolución. Además de la diseminación en un conjunto extenso de campos de conocimiento disciplinares e interdisciplinares, de nuevas y reconceptualizadas sub-disciplinas o áreas de interés. También entrado el nuevo siglo comienzan a aparecer signos de otras formas de relación entre las antropologías de ámbitos geopolíticos muy distintos, como la norteamericana, la europea y otras como la brasileña, la sudafricana, o la australiana. Se comenzaba a citar el papel renovador, el enriquecimiento que suponía el diálogo con estas otras tradicionales nacionales en Antropología. Hasta que, posteriormente, sería reseñable el proyecto surgido de la mano de autores como A. Escobar y Gustavo Lins Ribeiro, llamado Antropologías del mundo, ${ }^{12}$ que pretendía visibilizar la riqueza de aprendizajes que podían ofrecer las diferentes tradiciones nacionales en Antropología, dentro de sus particulares procesos históricos de conocimiento y desarrollo sociopolítico.

Y en cierto modo creo que puede decirse, que, llegado este punto del relato, ya nos encontraríamos en el contexto actual de los debates que explícita o implícitamente tratan de dirimir la identidad y el futuro de la disciplina. Constatada la necesidad de un uso más cauteloso respecto a sus utilidades en las estrategias culturalistas. ${ }^{13}$ Con una creciente consciencia de que, seguramente, el compromiso con la práctica etnográfica no basta para sostener una unidad disciplinar que precisa negociarse sobre la realidad heterogénea de antropologías existentes y posibles, y que tiene en el holismo ${ }^{14}$ su mejor posibilidad de un mínimo común para articularse ella misma y para establecer diálogos con otras ciencias. ${ }^{15}$

Volviendo la mirada de forma más específica al contexto español, hay que señalar que en general las reflexiones explícitas sobre la cuestión del futuro de la disciplina y su identidad son menos numerosas de lo que cabría esperar. Aunque es cierto que, como temática general, a menudo ha aparecido como marco de congresos y reuniones científicas. Las aportaciones en este sentido suelen hacerse eco

12 Lins Ribeiro, G.; Escobar, A.: Antropologías del mundo. Transformaciones disciplinarias dentro de sistemas de poder, México DF: CIESAS, 2009.

13 Marcus, G. E.: «The once and future ethnographic archive», History of the Human Sciences, 11 (4), 1998, pp. 49-63.

14 Rubel, P.; Rosman, A.: «The past and the future of Anthropology», Journal of Anthropological Research, 50 (4), 1994, pp. 335-343.

15 Harkin, M.: «Uncommon Ground: Holism and the Future of Anthropology», Reviews in Anthropology, 39 (1), 2010, pp. 25-45. 
de contextos de cambio generales, ${ }^{16}$ pero solo contadas excepciones abordan la cuestión desde una historización particular de la Antropología española, y mucho menos desde análisis que tengan en cuenta los procesos de institucionalización académica de la misma. ${ }^{17}$ De todas formas de manera reciente esto ha comenzado a cambiar, y parece que hay trabajos que incorporan esas reflexiones, situando a la antropología española en el contexto europeo, ${ }^{18}$ y desde hace bastantes años hay núcleos que están, desde su práctica, proponiendo nuevas formas de entender la disciplina y así encarar el futuro. ${ }^{19}$

\section{LAS RESPUESTAS A LA ENCUESTA RELATIVAS A LA IDENTIDAD Y EL FUTURO DE LA ANTROPOLOGÍA EN ESPAÑA}

Como se mencionaba al inicio, la intención de este texto era ofrecer de manera contextualizada un esbozo sobre las respuestas obtenidas en la encuesta que se realizó sobre la situación profesional y académica de la Antropología española, y que sirve de base para este número de la Revista Murciana de Antropología. Dicha encuesta contenía una pregunta de respuesta abierta, cuyo enunciado hacía referencia a la percepción del encuestado sobre la definición de la identidad de la disciplina, sus atribuciones académicas y profesionales, así como su futuro. Concretamente si el encuestado contempla la Antropología como una vía para formar egresados especializados en Antropología, o por el contrario, le parece más posible o positivo que complemente la formación de otras disciplinas y profesionales. Lógicamente dicho enunciado lleva implícito el cuestionamiento por los

16 Velasco, H.: «El futuro es el pasado: La Antropología como ciencia de la cultura y como ciencia del hombre», Anales de la Fundación Joaquín Costa, 11, 1994, pp. 123-130. SANMARTín, R.: «Futuro de la Antropología social», Anales de la Fundación Joaquín Costa, 11, 1994, pp. 145-154.

17 Seguramente el caso de Anta Félez sea el único: ANTA FÉLEZ, J. L.: «La antropología social española en los 80 como paradigma tardomoderno», Revista de Dialectología y Tradiciones Populares, 60 (2), 2005, pp. 5-27.

18 Horolets, A.; Heintz, M. \& Barrera, A.: European Anthropologies, Bergham Books, 2017. Giglia, A.; Garma, C. \& De Teresa, A. P. (comps.): ¿Adónde va la antropología?, México DF, Universidad Autónoma Metropolitana-Iztapalapa, 2007.

19 Ferrandiz, F.: Etnografías contemporáneas: Anclajes, métodos y claves para el futuro, Barcelona: Anthropos, 2011. Flores Martos, J. A.; Mariano, L.: «Nuevas definiciones de evidencia en la Medicina contemporánea: aportes desde la Antropología», Saude e Sociedade, 25 (1), 2016, pp. 43-56. Estalella, A.; Corsín, A.: «Asambleas al aire: La arquitectura ambulatoria de una política en suspensión», Revista de Antropología Experimental, 13, 2014, pp. 73-88. FERnÁNDEZ DE RotA, A.: «La cultura de los científicos de la cultura: notas para la genealogía de un concepto y una práctica», Revista de Antropología Experimental, 13, 2012, pp. 389-394. Diz, C.: «Objetos tirados, objetos sagrados. Reapropiación tecnológica de la bicicleta», en Revista de Antropología Experimental, 16, 2016, pp. 147-165. 
aspectos nucleares, que de manera breve, pretendían introducirse en la contextualización llevada a cabo en el apartado anterior. Preguntar por la definición de la disciplina y la relación con otras disciplinas, introduce de manera articulada varias cuestiones claves. Lo relativo a la interdisciplinariedad y a la gestión de la relación con otros ámbitos de conocimiento que siempre parecen fagocitar o ignorar los aportes antropológicos, la cuestión de la articulación de la propia diversidad de antropologías que deben buscar formas de articularse, y como no, la diferencia entre distintas trayectorias académicas e intelectuales que pueden encontrarse entre los antropólogos. Como bien señala uno de los colegas que se prestaron a responder la encuesta, Juan A. Flores (UCLM), la respuesta al cuestionario, la perspectiva implícita en ella, está sin duda condicionada por la propia trayectoria, y en ese sentido una de las cuestiones de conjunto que arroja el cuestionario es lo interesante que resultaría poder recabar este tipo de informaciones de manera más sistemática, la posibilidad incluso de realizar un estudio etnográfico sobre las prácticas científicas e instituciones de los antropólogos españoles. Pero esto nos alejaría de la intención de este trabajo, que pasa más por querer presentar un repertorio de las posiciones existentes, tratando de promover un diálogo y una necesaria reflexión de conjunto.

Pese a ser escasas las respuestas con las que contamos, sí que parece, al menos en la cuestión específica de la identidad y el futuro disciplinar, que las respuestas recabadas ofrecen un despliegue bastante representativo de los diferentes tipos de antropólogos y antropologías existentes en el espacio académico nacional (aunque ciertamente tendríamos un problema de género con la muestra recogida). En general diríase que existen grandes espacios de acuerdo, o al menos de no desacuerdo, pero también sería necesario señalar que precisamente parece que lo significativo de las diferencias podría hallarse en los matices. Algunos de los encuestados representan trayectorias académicas formadas en otras ramas más generales como la Historia o la Filosofía, para luego especializarse en la Antropología. Otro segmento algo más joven, ya presenta una formación específicamente antropológica, y un pequeño sector, muy interesante desde mi punto de vista, representa lo que yo entendería como autores que sin considerarse específicamente antropólogos, se mueven y desarrollan su trabajo teniendo como herramienta principal la inclusión del conocimiento antropológico. Todos con mayor o menor entusiasmo reconocen la capacidad potencial de la Antropología para ofrecer resultados en el diseño e implementación de formas planificadas de intervención social, aunque el énfasis en ello parece distinguir entre quienes, de manera más o menos clásica o actual, alegan en favor de la vigencia de un proyecto netamente antropológico, y quienes parecen aceptar sin problema alguno el entronque con marcos inter o transdisciplinares. También es muy interesante la distinción 
entre algunos encuestados que hacen referencia al marco de condiciones sociopolíticas e institucionales en el que deviene el contexto que atraviesa la disciplina, y quienes no hacen alusión alguna a la cuestión.

De manera más detallada, puede decirse que todos los encuestados muestran en sus respuestas concepciones sobre la identidad de la disciplina en las que priman entendimientos prácticos de la misma, que están ligados con el compromiso con la metodología etnográfica, pero en los que se pueden encontrar matices en torno al énfasis en la orientación aplicada o más académica (lógicamente todos parten de lo necesario de ambas líneas). Así, por ejemplo, A. Díaz de Rada (UNED) arranca de una definición disciplinar que, manteniendo continuidades con las concepciones digamos clásicas, se actualiza desde un énfasis en la unidad de las diferentes subdisciplinas y enfoques, resaltando lo distintivo de quizá la única forma de conocimiento biocultural. $\mathrm{Su}$ «flexibilidad teórica» y metodológica hace que lo esencial del conocimiento antropológico radique en su capacidad para analizar las prácticas humanas:

\begin{abstract}
«Sin embargo - especialmente a lo largo de las últimas décadas-, los antropólogos hemos llegado a comprender que tal diferencia conceptual entre "nosotros" y "los otros" se convierte en ficticia, una vez que aprendemos a mantener un adecuado extrañamiento de lo propio. Hoy la antropología rechaza toda forma de exotismo, pues nada es realmente externo a su verdadero objeto considerado del modo más general: la acción social humana situada en concretos entornos de práctica».
\end{abstract}

Por su parte, tanto D. Florido (U. de Sevilla) como A. Moncusí (U. de Valencia) hacen, quizá, más énfasis en la vinculación de este entendimiento interdisciplinar de la disciplina ligado con un enfoque más aplicado.

«Considero a la antropología como una práctica especialmente adecuada para la traducción intercultural, de ahí su posibilidad de aplicación a distintos campos: medicina y salud, educación, relaciones humano-ambientales, relaciones sociales (interétnicas, intergénero, entre grupos generacionales...), como modo de acceder a otros objetivos, preguntas y respuestas humanas, etc. otros campos tienen que ver con una demanda creciente, en términos sociales, pero también con consecuencias económicas, en relación con la cultura como patrimonio, el relato cultural sobre determinados colectivos y sus producciones culturales, para su difusión social. otras aplicaciones crecientes tienen que ver con la ciencia y la tecnología o las redes sociales virtuales, que se han convertido en canales e instrumentos de mediación de la experiencia social» (Florido).

«Entiendo que la Antropología tiene encaje laboral sobre todo en:

Cuestiones como servicios y diseño de políticas relacionadas con la diversidad étnica y cultural (migraciones, minorías étnica, interculturalidad...) en ámbito educativo, social y cultural. 
- Puestos profesionales dedicados al patrimonio (en especial el inmaterial).

- Cooperación al desarrollo internacional y desarrollo comunitario/local» (Moncusí).

En esta misma línea de primacía de definiciones prácticas de la disciplina, en los que los matices determinantes radican en la oscilación entre entendimientos más académicos o aplicados, resulta interesante también las aportaciones de autores que parecen enfocar el énfasis aplicado en el enriquecimiento de otras disciplinas/prácticas profesionales, que curiosamente ofrecen sus puntos de vista desde un conocimiento relacionado con la antropología de la salud. M. Moreno (U. Europea de Madrid), con un interesante doble bagaje, antropólogo y enfermero, aboga por aparcar los debates identitarios sobre la definición teórica de las disciplinas y centrarse en sus posibilidades prácticas de implementar análisis útiles (una posición similar se intuye en las respuestas de E. Perdiguero-UMHE- en otras partes del cuestionario, aunque de este particular, en concreto, se inhibió por no definirse como antropólogo):

«El pasado día 8 de mayo asistí a la celebración del día internacional de la enfermera organizado por el Consejo General de Enfermería donde participaban muy relevantes figuras de la profesión; para mi sorpresa el debate se centró en la identidad: ¿qué son las enfermeras? ¿Cómo nos definimos? ¿Cómo nos ve la sociedad? Etc. Yo me decía ¿pero esto no lo tenemos aún superado? Hace casi 30 años Collière ya criticaba por estéril este exceso de endogamia de la profesión y señalaba que más que interrogarse sobre lo que somos hay que interrogarse sobre lo que hacemos. Comparto esta posición que puede trasladarse perfectamente a los antropólogos. Hay que involucrarse en las demandas sociales y el reconocimiento, y la identificación profesional vendrán por sí solo. [...] Ya hay muchos antropólogos que están trabajando en ellos y ese creo que es el camino» (Moreno).

O las respectivas respuestas de L. Moro (USAL) o AM. Nogué (UMHE), que ponen en juego decididas defensas en pro de las superación de unos límites disciplinares que vendrían más impuestos por lógicas institucionales o de producción del conocimiento:

«Principalmente a la cuestión acerca de ¿qué es la Antropología y cómo nos definimos como antropólogos? A esas preguntas yo añado una reflexión que creo, es también muy importante ¿dónde van a trabajar esos antropólogos que nosotros pensamos formar? Incluso en el caso de que se ofertaran plazas en el ámbito docente ¿es esa suficiente justificación? Efectivamente, como planteáis, desde la antropología tenemos relación con multitud de campos de trabajo (educación, derecho, salud, criminología...) lo que me lleva a preguntar si debemos formar antropólogos o contribuir a la formación de los psicólogos, médicos, abogados... desde una disciplina holística como es la antropología, que permite y posibilita una comprensión y un conocimiento integrado de las diferentes dimensiones en que se desenvuelve la vida de las personas» (Moro). 
«De hecho siempre me defino como profesor universitario y casi nunca como antropólogo. Tengo serias dificultades para hablar de disciplinas científicas y por esta razón, recurro al global de ciencias sociales y humanas o humanidades. Esto me lleva a pensar que las disciplinas, al menos en el mundo universitario europeo que es el que más y mejor conozco, responden a un parcelamiento académico que, en absoluto, ni busca ni pretende la unidad de la ciencia ni del conocimiento.

De lo anterior se deduce que siempre haya tenido una posición muy crítica respecto a las posibilidades laborales de la antropología social como disciplina que se diferencie de profesiones más consolidadas. Las características propias del quehacer socio-antropológico (entre otras, el tiempo que requiere y el compromiso con un pensamiento crítico) dificulta mucho que, por las feroces características neo-liberales del mercado laboral actual, resulte una profesión atractiva para el sector privado y, dado el corporativismo de profesiones ya consolidadas en las administraciones, pues que pueda hacerse un hueco en el sector público más allá de uno puramente testimonial» (Nogué).

En cuanto al cuestionamiento por la percepción sobre el futuro de la disciplina, al margen de los tonos más o menos optimistas o pesimistas que pueden encontrarse en las perspectivas de los encuestados, cabe sobre todo reseñar que es en este punto, al elaborar la concatenación reflexiva entre la caracterización de la disciplina y sus posibilidades a futuro, donde surgen posiblemente los aspectos más jugosos, haciendo referencia a distintas cuestiones de contexto de mucho interés, que van desde el contexto socioeconómico que atraviesa las universidades en la actualidad, hasta las racionalidades políticas hegemónicas, pasando por la conformación institucional del conocimiento científico en general y de la antropología en particular.

En este sentido M. Moreno señala dos cuestiones presentes en el debate social de actualidad, el arrinconamiento de las Humanidades en nuestras universidades, y lo que se percibe como un déficit de ética en las mismas:

«Trasladando los argumentos expresados a la actualidad, creo que el principal problema que tiene la disciplina está en el arrinconamiento progresivo de las humanidades. Hace unos diez años presenté en un congreso internacional un estudio sobre la formación en diversidad cultural (en un periodo de fuerte flujo migratorio en España) y allí ofrecía datos que evidenciaban la poca atención que se estaba prestando a la necesaria educación en la diversidad para afrontar esta nueva realidad: escasez de asignaturas en la universidad sobre la temática y de igual manera insuficiente programas formativos en el ámbito sanitario. Unos cuantos años después cabría preguntarse ¿cómo está ahora la situación? ¿Han cambiado los factores sociales que inciden en el eje educación-diversidad? ¿Ha aportado algo el Plan Bolonia? Aún sin disponer de datos suficientes, creo que la enseñanza en diversidad cultural no ha avanzado nada e incluso, por algunas informaciones de que dispongo, ha retrocedido. En algunas universidades aquellos escasos programas sobre diversidad cultural han pasado a diluirse en otras asignaturas o han pasado a formato online. El retroceso de las humanidades, a nivel general, afecta negativamente a los enfoques educativos que 
se alejan del paradigma dominante que prioriza la educación centrada en el mercado. Lo mismo puede decirse en el ámbito sanitario donde los recortes sanitarios se han llevado por delante los pocos programas formativos que había sobre diversidad cultural.

Paralelamente, la actualidad nos pone ante un problema que los antropólogos no deben eludir: la falta de ética en la universidad (como señalaba Anta) no han hecho más que crecer. Los últimos acontecimientos mediáticos nos ponen ante una duda ¿son casos aislados o son la punta del iceberg? Pienso que es un problema estructural y que salen a la luz porque hoy la sociedad ya no permite prácticas antaño toleradas» (Moreno).

A su vez A. M. Nogué parece enfatizar una percepción positiva del abandono de las pretensiones disciplinares más clásicas o convencionales, en pos de la transformación en una estrategia de conocimiento con múltiples ramificaciones que puede complementar muchas otras y aplicarse en múltiples ámbitos sin necesidad de la estructura, por ejemplo, de un Grado propio:

\begin{abstract}
«Por el contrario, estoy plenamente convencido de que la antropología social podría desarrollar muchísimo mejor su función social de utilidad (en los términos que hoy se considera esa 'utilidad') y mostrar todo su potencial intelectual como disciplina transformadora, si se entendiese como complemento formativo de otras profesiones. Creo que la antropología social encuentra su mayor sentido si lleva a profesionales y a futuros profesionales a reflexionar sobre sobre los fundamentos de sus propias ideas. La experiencia acumulada como licenciatura de segundo ciclo así lo demuestra. Siempre he pensado que el empecinamiento obsesivo por transformar la antropología social en un grado Bolonia, respondió más a una estrategia que evitase que la disciplina se viera relegada a una situación de inferioridad académica y, a la postre, a una merma de la relevancia pública e intelectual de los profesores, que a un convencimiento real de la 'utilidad' laboral de la disciplina para los futuros egresados» (Nogué).
\end{abstract}

Por su parte, C. Diz (U. de A Coruña), seguramente el perfil más «joven» entre los encuestados, posiblemente sea el que ofrezca en su respuestas algunas claves más actuales respecto a la cuestión del futuro de la disciplina. Sus respuestas muestran en la misma medida un reconocimiento amplio de las dificultades que el contexto social y económico dispone para el tipo de conocimiento que constituye la Antropología:

«No es fácil responder a esta pregunta, y menos en un contexto como el actual. Por un lado, todo parece indicar que el descalabro que este mundo trae consigo -a nivel económico, social, político, moral, climático, etc.- pone de manifiesto cuánto de importante es la antropología en un tiempo tan convulso en el que escuchar al otro y entenderse a uno mismo se ha vuelto tan urgente. A nivel académico, las políticas de austeridad y la lógica neoliberal que han convertido a la Academia en un nicho de neoliberalismo, individualismo, hiper-competitividad e hiper-burocratización, hacen que sea difícil animar a las nuevas generaciones a emprender una carrera investigadora, cuando miles y miles de compa- 
ñeros y compañeras han tenido que emigrar en busca de oportunidades en este campo. A su vez, no hace falta irse muy lejos para encontrar proyectos nuevos, como por ejemplo el de «Antropología 2.0», que buscan huecos para la antropología en casi cualquier área del mercado laboral, habiendo entendido que la salida académica para la antropología es solo una, y por desgracia -a día de hoy- solo al alcance de unos pocos».

Y una visión optimista respecto a las posibilidades de la disciplina, en este caso parte de una percepción mucho más flexible y desprejuiciada sobre los medios que debe utilizar la disciplina para retomar los grandes debates científico y también sociales, que pasa, como no, por una presencia en el medio educativo que parece más urgente que nunca:

«En este sentido, creo que se ha vuelto imprescindible comunicar más y mejor la antropología, ponerla en valor en un contexto en el que podría ser de gran utilidad, sacarla fuera de la academia, airearla, «popularizarla». Sin duda, penetrar en otras disciplinas (como se hace en la educación, por ejemplo) es importante para llevar a cabo esta misión pedagógica de explicar la antropología y llevarla a cuantos más escenarios mejor. Pero la antropología ha de reivindicarse también como lo que en el fondo es, una disciplina única y singular, con capacidad de tener voz en la realidad, de contarla y también de transformarla. Pero, en cierto modo, esto desafía la lógica endogámica y el escenario-burbuja en el que vive la universidad -a menudo encerrada en sí misma, en sus lógicas de producción y reproducción, y separada de aquellos en cuyo nombre dice hablar; por eso es importante extender la antropología más allá de los campus universitarios: dar la batalla también en los institutos, y por qué no en las escuelas, tratar de implantar en los planes de formación al menos una asignatura de antropología, y también, defender a los egresados/as en antropología para que les sea reconocida (porque no siempre lo es) su formación en antropología y su capacidad de enseñar materias tan afines y relacionadas con cuestiones como el género, la sexualidad, los 'valores cívicos', la cultura, etc.».

Por último creo que también merecían una atención más específica las respuestas de J. A. Flores. Al igual que la mayor parte de las anteriores, aunque tal vez de una manera más clara, en su abordaje sobre la cuestión del futuro de la disciplina, Flores apuesta por la articulación de estrategias de conjunto que vertebran la doble posibilidad del trabajo con la consolidación y crecimiento del ámbito específicamente antropológico, tanto a nivel académico como profesional, al tiempo que también propugna la profundización en la colaboración en la formación de otras disciplinas y la investigación-aplicación interdisciplinar.

«Creo que debemos continuar nuestra labor docente y lucha por expandir nuestra influencia y radio de acción formando en Antropología - como ciencia auxiliar y complementaria- a estudiantes y futuros profesionales de otras disciplinas: enfermeras, trabajadoras sociales, educadores sociales, terapeutas ocupacionales, etc. Esta es una vía irrenunciable para tener presencia e influencia en la realidad de la intervención en el campo de lo social 
y de la salud. Además en ocasiones despertamos el interés, y la formación o vocación posterior de estos profesionales hacia la Antropología como su futura dedicación complementaria o principal.

Al mismo tiempo, debe priorizarse y mantenerse la formación específica en Antropología en grados, másteres oficiales y doctorados, ya que solo formando antropólogos podemos participar en el desarrollo teórico específico y en el crecimiento de la Antropología en el siglo XXI».

Lo que sucede es que la respuesta de Flores es quizá la que parte de una conciencia más clara en cuanto a realizar un diagnóstico de los procesos que han conducido hasta la situación actual, y a partir de ahí cuáles pueden ser los pasos a seguir. Y en ese sentido, su respuesta es la única que pone sobre la mesa un debate que ha venido siendo polémico en el seno de la disciplina desde hace mucho tiempo.

«Tanto la identidad de la Antropología en España, como su evolución y eventual desarrollo en el futuro, pasa a mi juicio por algunas cuestiones que trataré de resumir del modo más telegráfico posible. No obstante, espero que esa breve enunciación no nos confunda sobre la complejidad y radical prioridad que tienen estos retos para nuestra supervivencia como profesionales de este oficio:

No podemos permitirnos el lujo de repetir los errores del pasado como colectivo: los antropólogos somos los principales responsables de lo que nos pasa -o nos deja de pasara los antropólogos. Si no llegamos a unos consensos básicos, una organización unitaria -Colegio Profesional Español de Antropólogos- y a unos pactos de no agresión entre espacios de poder y territorios, continuaremos siendo fagocitados por nuestros colegas -nuestros «primos más listos»- de otras disciplinas: sociólogos, psicólogos, pero también enfermeros o trabajadores sociales.

Sin ese Colegio que sea nuestro órgano de expresión y de presión será difícil -por no decir imposible- cambiar la realidad actual. Una realidad donde los antropólogos seguimos sin estar en las comisiones de Ciencias Sociales, pero también de Humanidades, tanto de las instituciones de Docencia e Investigación nacionales, regionales, locales, como en las de las Fundaciones y organismos privados que otorgan premios, becas, ayudas de investigación, etc. Seguimos siendo evaluados por colegas con alta competencia en Psicología, Economía, Derecho o Ciencias Políticas, y si tenemos suerte por alguien de Sociología».

\section{A MODO DE CONCLUSIÓN: FUTUROS ANTROPOLÓGICOS}

Me parece que este apartado final, y cierre del texto, se debería aprovechar con una doble finalidad. Primero para ofrecer algún tipo de recapitulación sobre la cuestión de los retos que el futuro deparará a la Antropología (tal y como se ha presentado y se desprende de las respuestas a la encuesta realizada). Y en segundo lugar, también para ofrecer un breve posicionamiento personal sobre la men- 
cionada cuestión, que en este caso discurrirá en torno a la posibilidad de incorporar otra acepción diferente respecto a la idea de futuro. En relación a la cuestión de las respuestas a la encuesta y la percepción sobre el futuro de la Antropología en España, no podría añadir ni quitar una sola coma de lo expresado por mis compañeros, reconozco en sus palabras muchas de las antropologías que se desarrrollan a dia de hoy en eso que a veces definimos como disciplina. Expresadas de distinta manera, comparto y entiendo la valía de todas las vías de desarrollo apuntadas: la consolidación de un espacio académico propio actualizado, la intensificación de los lazos cooperativos con otras disciplinas, la lucha por la constitución de un espacio de desarrollo profesional que fortalezca las formas de aplicación, y como no, la lucha por los grandes debates y el apoyo en otras estrategias comunicativas y divulgativas más abiertas que incluyan pasos para acceder a los curriculums educativos. La pregunta que me hago al contemplar ese despliegue de opciones potenciales, es si como colectivo, la disciplina está dispuesta a desarrollar una serie de acciones estratégicas (en el sentido, por ejemplo, expresado por Flores) que permitan conseguir algunas de estas metas. Entiendo también que este interrogante, por su complejidad, entraña nuevos debates.

En cuanto a la cuestión del futuro, comparto una idea que forma parte de la tradición disciplinar desde sus orígenes, y que de manera más reciente ha ido redefiniéndose y ganando pujanza. Por supuesto no es una opción única que en sí misma solvente las dificultades y retos que encara la disciplina, pero al menos, desde mi perspectiva y coincidiendo con la de otros autores, aparece como una opción muy interesante. Dicha opción, en esencia y expresada de manera muy básica, consiste en tratar de aprovechar el acervo de conocimientos sobre la naturaleza biocultural de los seres humanos y sus sistemas de prácticas, así como sobre sus desarrollos sociales y tecnológicos pasados y presentes, para intentar ofrecer un conocimiento especulativo sobre las posibles consecuencias y efectos futuros de los procesos sociales, tecnológicos, políticos y económicos extremádamente críticos y disruptivos que parece que nuestras sociedades se disponen a atravesar. Esta posibilidad ya aparecía, por ejemplo, en la reflexión sobre el futuro disciplinar de Cyril Belshaw, ${ }^{20}$ que animaba a desarrollar dicha estrategia en las nuevas pautas de formación académica que se deberían desarrollar para llevar a cabo una transformación. De hecho la ineludible irrupción de los nuevos desarrollos en genética, biotecnología, tecnologías de la información y la comunicación, la robótica o la inteligencia artificial, han ido haciendo que, sobre todo en la antropología de habla inglesa (pero es una tendencia que se extiende rápidamen-

20 Belshaw, C.: «Retos de la Antropología social y cultural en el futuro», Revista Internacional de Ciencias Sociales. Tendencias de la Antropología, 116, 1988, pp. 201-212. 
te), se vaya centrando, con cada vez mayor énfasis, en el análisis de los procesos científicos y tecnológicos, y lo que estos implican como tendencias emergentes, como realidades que se deben leer en clave de presente y de futuro. ${ }^{21}$

En mi caso no considero que el vínculo cuasi genético del pensamiento antropológico con el cuestionamiento por el tiempo, en el marco de su constitución moderna, conlleve la necesidad de que la antropología deba instituirse como parte de una hipotética «futurología»,22 pero sí que considero que se trata de un área de desarrollo para nuevas estrategias de conocimiento y divulgación relacionadas con lo apuntado durante el trabajo. Me parece, por ejemplo, una evidencia positiva a favor de esta idea, que recientemente una voz heterodoxa pero de reconocido rigor como la del escocés T. Ingold, ${ }^{23}$ haya defendido el valor de esa veta especulativa en la tradición de conocimiento antropológico. Por supuesto, como decía, no parece que esta ni otra opción sea la solución en sí mismas a todas las dificultades a considerar. A ese respecto parece que por ahora solo nos cabe comenzar a desarrollar estrategias de divulgación y reafirmación creativa de lo capitalizado, tal y como señala el inspirador P. Stoller en su llamado a una antropología de «guerrilla» recuperando las palabras de B. Kapferer:

\begin{abstract}
«Most of the guerilla anthropologists I've met understand, I think, that the old colonialist way of solving social problems or understanding the world doesn't work anymore. Our various systems of politics, economics and scholarship have become ineffectual and counter-productive. In this context, the guerilla approach to anthropology is perfectly suited to living in, coping with and understanding contemporary social worlds. In my work as public blogger I attempt to present an anthropological perspective on contemporary social and political issues. In that work I often extend the wisdom of the aforementioned Songhai people (an exercise in guerilla anthropology) to the pragmatic analysis of our social, political, and existential issues» (Stoller). ${ }^{24}$
\end{abstract}

21 Escobar, A.: «Welcome to Cyberia: Notes on the Anthropology of Cyberculture», Current Anthropology, 35 (3), 1994, pp. 211-231. Fischer, M. J.: Emergent Forms of Life and the Anthropological Voice, Duke University Press, 2003. Rabinow, P.; Marcus, G. E.; Faubion, J. D. \& REEs, T. (eds.): Designs for an Anthropology of the Contemporary, Duke University Press, 2008.

22 Strzelecka, C.: «Anticipatory anthropology-anthropological future study», Prace Etnograficzne, 41(4), 2013, pp. 261-269.

23 Ingold, T.: Ambientes para la vida Conversaciones sobre humanidad, conocimiento y antropología, Montevideo: Trilce, 2012.

24 <ttps://paulstollersblog.com/2018/08/05/a-guerilla-anthropologist-looks-to-the-future/>, Blog de Paul Stoller: «A Guerrilla Anthropology», publicado el 05/08/18. Consultado el 03/09/18. 


\section{BIBLIOGRAFÍA}

ANTA FÉLEZ, José L:, «La antropología social española en los 80 como paradigma tardomoderno», Revista de Dialectología y Tradiciones Populares, 60 (2), 2005, pp. 5-27.

AKBAR, Ahmed; SHORE, Chirs (eds.): The Future of Anthropology: its Relevance to the Contemporary World, Londres: Athlone, 1995.

BELSHAW, Cyril: «Retos de la Antropología social y cultural en el futuro», Revista Internacional de Ciencias Sociales. Tendencias de la Antropología, 116, 1988, pp. 201-212.

CLIFFORD, James: «Looking in Several Ways: Anthropology and Native Heritage in Alaska», Current Anthropology, 45 (1), 2004, pp. 5-30.

CLIFFORD, James; MARCUS, George: Writing Culture. The Poetics and Politics of Ethnography, Berkeley: University of California Press, 1986.

COMAROFF, John: «The End of Anthropology, Again: On the Future of an In/Discipline», American Anthropologist, 112 (4), 2010, pp. 524-538.

DIZ, Carlos: «Objetos tirados, objetos sagrados. Reapropiación tecnológica de la bicicleta», Revista de Antropología Experimental, 16, 2016, pp. 147-165.

ESCOBAR, Arturo: «Culture, Practice and Politics: Anthropology and the Study of Social Movements», Critique of Anthropology, 12 (4), 1992, pp. 395-432.

ESCOBAR, Arturo: "Welcome to Cyberia: Notes on the Anthropology of Cyberculture», Current Anthropology, 35 (3), 1994, pp. 211-231.

ESTALELLA, Adolfo; CORSÍN, Alberto: «Asambleas al aire: La arquitectura ambulatoria de una política en suspensión», Revista de Antropología Experimental, 13, 2014, pp. 73-88.

FERNÁNDEZ DE ROTA, Antón: «La cultura de los científicos de la cultura: notas para la genealogía de un concepto y una práctica», Revista de Antropología Experimental, 13, 2012, pp. 389-394.

FERNÁNDEZ DE ROTA Y MONTER, José Antonio: Una etnografía de los antropólogos en EE.UU. Consecuencias de los debates posmodernos, Madrid: Akal, 2012.

FERRÁNDIZ, Francisco: Etnografias contemporáneas: Anclajes, métodos y claves para el futuro, Barcelona: Anthropos, 2011.

FISCHER, Michael J.: Emergent Forms of Life and the Anthropological Voice, Duke University Press, 2003.

FLORES MARTOS, Juan A.; MARIANO, Lorenzo: «Nuevas definiciones de evidencia en la Medicina contemporánea: aportes desde la Antropología», Saude e Sociedade, 25 (1), 2016, pp. 43-56. 
GIGLIA, Ángela; GARMA, Carlos \& DE TERESA, Ana Paula, (comps.): ¿Adónde va la antropología?, México DF, Universidad Autónoma Metropolitana-Iztapalapa, 2007.

HARKIN, Michael: «Uncommon Ground: Holism and the Future of Anthropology», Reviews in Anthropology, 39 (1), 2010, pp. 25-45.

HOROLETS, Anna; HEINTZ, Monica \& BARRERA, Andrés: European Anthropologies, Bergham Books, 2017.

HYMES, Dell (ed.): Reinventing Anthropology, New York: Vintage Books, 1970.

INGOLD, Tim: Ambientes para la vida Conversaciones sobre humanidad, conocimiento y antropología, Montevideo: Trilce, 2012.

KUHN, Thomas: La estructura de las revoluciones cientificas, México DF: Fondo de Cultura Económica, 2004.

LINS RIBEIRO, Gustavo; ESCOBAR, Arturo: Antropologías del mundo. Transformaciones disciplinarias dentro de sistemas de poder, México DF: CIESAS, 2009.

MARCUS, George E.: «The once and future ethnographic archive», History of the Human Sciences, 11 (4), 1998, pp. 49-63.

MARCUS, George; FISCHER, Michael: Anthropology as Cultural Critique. An Experimental Moment in the Human Sciences, Chicago: Chicago University Press, 1986.

NEEDHAM, Rodney: "The future of Social Anthropology: disintegration or metamorphosis», Anniversary Contributions to Anthropology: twelve essay, Leiden: Brill, 1970.

ONG, Aiwa; COLLIER, Stephen (eds.): Global Assemblages: Technology, Politics, and Ethics as Anthropological Problems, Nueva York: Blackwell, 2004.

RABINOW, Paul; MARCUS, George E.; FAUBION, James D. \& REES, Tobias, (eds.): Designs for an Anthropology of the Contemporary, Duke University Press, 2008.

RUBEL, Paula; ROSMAN, Abraham: «The past and the future of Anthropology», Journal of Anthropological Research, 50 (4), 1994, pp. 335-343.

SANMARTÍN, Ricardo: «Futuro de la Antropología social», Anales de la Fundación Joaquín Costa, 11, 1994, pp. 145-154.

SCHEPER-HUGHES, Nancy: «The Primacy of the Ethical Propositions for a Militant Anthropology», Current Anthropology 36 (3), 1995, pp. 409-440.

STRZELECKA, Celina: "Anticipatory anthropology-anthropological future study», Prace Etnograficzne, 41 (4), 2013, pp. 261-269.

VELASCO, Honorio: «El futuro es el pasado: La Antropología como ciencia de la cultura y como ciencia del hombre», Anales de la Fundación Joaquín Costa, 11, 1994, pp. 123-130. 
